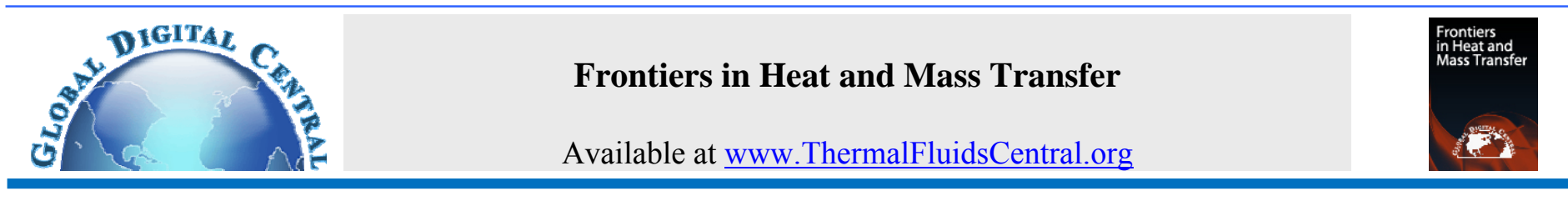

\title{
VARIABLE THERMAL CONDUCTIVITY INFLUENCE ON HYDROMAGNETIC FLOW PAST A STRETCHING CYLINDER IN A THERMALLY STRATIFIED MEDIUM WITH HEAT SOURCE/SINK
}

\author{
P. Sreenivasulu ${ }^{\mathrm{a}, *}$ T. Poornima ${ }^{\mathrm{b} \dagger}$, N. Bhaskar Reddy ${ }^{\mathrm{c},}$ \\ a Department of Mathematics(HAS), Sri Venkateswara College of Engineering and Technology, Chittoor, A. P., 517127, India. \\ ${ }^{b}$ School of Advanced Sciences, VIT University, Vellore, T.N. , 600127, India. \\ ${ }^{c}$ Department of Mathematics, Sri Venkateswara University, Tirupati, A. P., 517502, India.
}

\begin{abstract}
This paper examines the variable thermal conductivity influence on MHD flow past a thermally stratified stretching cylinder with heat source or sink. The governing partial differential equations of the flow field are converted to a system of non-linear coupled similarity ordinary differential equations. Employing Shooting technique followed by Runge-Kutta method, the system is solved numerically. The effects of the various physical parameters countered in the flow field on the velocity, temperature as well as the skin friction coefficient and the rate of heat transfer near the wall are computed and illustrated graphically.

Keywords: Variable Thermal conductivity; Radiative heat transfer; Magneto hydrodynamic; Thermal stratification; Viscous flow.
\end{abstract}

\section{INTRODUCTION}

Recent era is transferring of heat through lubricants due to stretching cylinder plays major role in the extrusion process of fibre technology, making of polymer sheets and plastic films, manufacturing paper, glass blowing, spinning of metals and plastic films, etc,. Influence of MHD on nanofluid past non-linear stretching sheet with heat absorption was discussed by Sreenivasulu et al. (2015). Over an exponentially stretching sheet, the slip effects on MHD flow is analyzed by Hayat et al. (2016). Swati and Anuar (2012) explained in detail regarding the convection flow past stretching cylinder. Analytically, a study past exponentially stretching sheet with thermal radiation effects on MHD flow is made by Kalpna and Gupta (2016). Subba Rao and Nagendra (2015) examined the radiation effects on Oldroyd-B nanofluid past a stretching sheet. Rasekh (2012) analyzed the heat source effect due to a stretching circular cylinder. Salahuddin et al. (2016) investigated by Keller box method on MHD Pseudo-plastic flow past a stretching cylinder. Sreenivasulu and Bhaskar Reddy (2013) investigated the stagnation point flow past a stretching porous sheet using Lie group analysis.

Stratification is due to the density variation in the fluid which gives rise to the temperature variations. So, that it is an important concept that one has to consider in studying the flow field. Best examples for this type of medium are congested containers, stratified ocean environment and biological chambers with heat blown walls. Flows with thermal stratification such as buoyant flow systems, Lake Thermohydraulics, volcanic flows and thermal treatment involved in the industrial processes. Stratification of the medium may arise due to a temperature variation, which gives rise to a density variation in the medium. This is known as thermal stratification and usually arises due to thermal energy input into the medium from heated bodies and thermal sources. The flow due to a thermally blown surface immersed in a stable stratified viscous fluid has been investigated experimentally. Deka and Neog (2009) explained in detail about the thermal stratification on an unsteady flow. Combined thermal and concentration stratifications on a Jeffrey fluid was discussed by Hayat et al. (2014). Nirmal (2016) analyzed the transient natural convection on a nanofluid with thermal stratification. Thermal stratification past an infinite cylinder was examined by Deka and Paul (2012). Kishore et al. (2010) investigated the effects of thermal stratification and dissipation along an infinite accelerated vertical plate.

Magnetic field effects on a conducting fluid received good attention from researchers. This is because hydromagnetic flow and heat transfer have become more important in industrially. For example, many metallurgical processes such as drawing, annealing and tinning of copper wires involve cooling of continuous strips or filaments by drawing them through a quiescent fluid. Controlling the rate of cooling in these processes can affect the properties of the final product. This can be done by using an electrically-conducting fluid and applying a magnetic field. Rushi Kumar (2015) explained the diffusion and radiation effects on plate in the presence of magnetic field. Mahapatra et al. (2014) analyzed the natural convection upon a horizontal flat plate with magnetic effects. The influence of heat absorption and source on MHD past stretching sheet in a non-Darcian medium was examined by Ibrahim and Shankar (2016). Poornima et al. (2016) analyzed the MHD mixed convective flow past a circular cylinder with chemical reaction effects. MHD convective flow past a linear stretching sheet using Lie symmetry analysis with thermal stratification was conducted by Rosmila et al. (2012).

The various physical properties of a fluid flow need not to be

*Corresponding author.Email: poornima.t@vit.ac.in 
constant always, it may vary with respect to the time or temperature. Because of the temperature differences, there arises a physical property change. Slip flow of Casson fluid with variable fluid physical property under radiation was studied by Poornima et al. (2015). Bhaskar Reddy et al. (2014) investigated the impact of variable thermal conductivity past a stretching sheet. Subba Rao et al. (2016) studied the radiation effects past variable porosity with slip. On the vertical stretching sheet, the thermal buoyancy and variable thermal conductivity with heat source is studied by Abel et al. (2009). Ojjela et al. (2016) studied the varying thermal conductivity impact on UCM fluid. Rangi and Ahmad (2012) analyzed the boundary layer flow past a stretching cylinder and heat transfer with variable thermal conductivity.

With the above awareness, it is seen that the interaction of heat transfer with variable thermal conductivity in an electrically conducting fluid past a stretching cylinder in a thermally stratified medium has received a little attention. Hence, the object of this paper is to study the effect of variable thermal conductivity on MHD boundary layer flow past a stretching cylinder in a thermally stratified medium with heat generation or absorption.

\section{MATHEMATICAL ANALYSIS}

Consider a steady axi-symmetric flow along a continuously stretching circular cylinder. The fluid is electrically conducting, so that the magnetic field is applied transversely. The cylinder is completely immersed in thermally stratified saturated medium with a variable ambient temperature $T_{\infty}(\mathrm{x})$, where $T_{w}(\mathrm{x})>T_{\infty}(\mathrm{x})$. The magnetic Reynolds number is assumed to be very small, so that the induced magnetic field is negligible. Figure 1 below gives the brief description of flow considered.

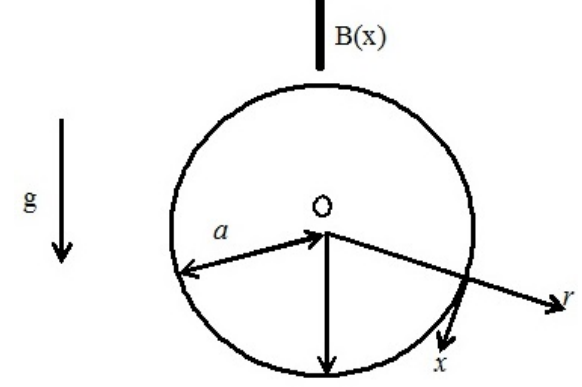

Fig. 1 Physical model and coordinate system

The Prandtl's boundary layer equations governing the flow under Boussinesq's approximation are

$\frac{\partial(r u)}{\partial x}+\frac{\partial(r v)}{\partial r}=0$

$u \frac{\partial u}{\partial x}+v \frac{\partial u}{\partial r}=\frac{v}{r} \frac{\partial}{\partial r}\left(r \frac{\partial u}{\partial r}\right)+g \beta\left(T-T_{\infty}\right)-\frac{\sigma B_{0}^{2}}{\rho} u$

$u \frac{\partial T}{\partial x}+v \frac{\partial T}{\partial r}=\frac{1}{r} \frac{\partial}{\partial r}\left(\alpha r \frac{\partial T}{\partial r}\right)+\frac{Q_{0}}{\rho C p}\left(T-T_{\infty}\right)$

The boundary conditions for the velocity and temperature fields are $u=U(x), \quad v=0, \quad T=T_{w}(x) \quad$ at $\quad r=R$

$u \rightarrow 0, \quad T \rightarrow T_{\infty}(x) \quad$ as $\quad r \rightarrow \infty$

An equilibrium position is maintained between the convection fluid and the medium with respect to temperature.

For liquid metals, the thermal conductivity $\alpha$ varies linearly with temperature in the range from $0^{\circ} \mathrm{F}$ to $400^{\circ} \mathrm{F}$. Therefore, we assume $\alpha$ as $\alpha=\alpha_{\infty}(1+\varepsilon \theta)$

where $\varepsilon(\leq 1)$ is the thermal conductivity parameter.

To get similarity solutions of equations (1) - (3) subject to the boundary conditions (4), we introduce the following similarity transformations.

$$
\begin{aligned}
& \eta=\frac{r^{2}-R^{2}}{2 R} \sqrt{\frac{U}{v x}}, \quad \psi=\sqrt{U v x} R f(\eta), \quad \theta=\frac{T-T_{\infty}}{T_{w}-T_{0}}, S=\frac{c}{b}, \\
& \gamma=\sqrt{\frac{v L}{U_{0} R^{2}}}, \lambda=\frac{g \beta L}{U_{0}^{2}}, M=\frac{\sigma B_{0}^{2} L}{\rho U_{0}}, \operatorname{Pr}=\frac{v}{\alpha_{\infty}}, Q=\frac{Q_{0} L}{\rho C p U_{0}} \\
& U(x)=\frac{U_{0} x}{L}, T_{w}(x)=T_{0}+b(x / L), T_{\infty}(x)=T_{0}+c(x / L)
\end{aligned}
$$

In view of equation (6), equations (1), (2) and (3) take the following dimensionless form.

$(1+2 \gamma \eta) f^{\prime \prime \prime}+2 \gamma f^{\prime \prime}+f f^{\prime \prime}-f^{\prime 2}+\lambda \theta-M f^{\prime}=0$

$(1+2 \gamma \eta) \theta^{\prime \prime}+2 \gamma \theta^{\prime}+\varepsilon\left[(1+2 \gamma \eta)\left(\theta^{\prime 2}+\theta \theta^{\prime \prime}\right)+2 \gamma \theta \theta^{\prime}\right]=\operatorname{Pr}\left(f^{\prime} \theta+S f^{\prime}-f \theta^{\prime}-Q \theta\right)$

where prime denotes the differentiation with respect to $\eta$.

The corresponding boundary conditions are

$f^{\prime}(0)=1, \quad f(0)=0, \quad \theta(0)=1-S$

$f^{\prime}(\infty) \rightarrow 0, \quad \theta(\infty) \rightarrow 0$

The engineering quantities of our curiosity in this study are the skin friction or the shear stress coefficient $C_{f}$ and the local Nusselt number $N u_{x}$, which are defined as

$\tau_{w}=\mu\left(\frac{\partial u}{\partial r}\right)_{r=R}, \quad q_{w}=-k\left(\frac{\partial T}{\partial r}\right)_{r=R}$

Using equation (9), the skin friction coefficient and local Nusselt number can be expressed as

$\frac{1}{2} \sqrt{\operatorname{Re}_{x}} C_{f}=f^{\prime \prime}(0), \frac{N u_{L}}{\sqrt{\operatorname{Re}_{x}}}=-\theta^{\prime}(0)$

where $\operatorname{Re}_{x}=\frac{U x}{v}$ is the local Reynolds number.

\section{NUMERICAL ANALYSIS}

A closed form solution is not possible for the equations (7) - (8) and boundary conditions (9), as these are coupled and highly non-linear boundary value problem. It is employed shooting technique to convert the BVP into IVP and then the IVP is solved numerically by RungeKutta method. In shooting method, $\eta_{\infty}$ ( maximum $\eta$ for which $\theta(\eta)$ $\rightarrow 0$ as $\quad \eta \rightarrow \infty)$ has to be shot first. For that the apt presumption $\theta$ '(0) is required so that the condition at the other end will be satisfied. To seek the missing $\theta^{\prime}(0)$, secant iterative method is applied.

After shooting the $\eta_{\max }$, it was chosen in such a way that further changes in it showed little changes (constant till $10^{-5}$ ) in the values of $\theta^{\prime}(0)$ vis- a- vis boundary condition $\theta(\eta) \rightarrow 0$ as $\eta \rightarrow \infty$ is satisfied, the initial conditions were determined.

With an effective step size $\Delta \eta=0.05$ and a convergence criterion of $10^{-5}$, a grid independent Runge - Kutta study was carried out by fixing $\eta_{\infty}=10$ (where it reaches the free stream velocity) to examine the solution in the quest for their optimization.

The shear stress coefficient and heat transfer rate at the wall are seen proportional to $f^{\prime \prime}(0)$ and $-\theta^{\prime}(0)$, respectively. Their numerical computations are portrayed using graphs.

\section{RESULTS AND DISCUSSION}

In order to get a physical insight into the problem, a representative set of numerical results is shown graphically in figures $2-17$, to illustrate the influence of governing parameters viz., $\gamma$ is the curvature parameter, $\lambda$ is the mixed convection parameter, $M$ is the magnetic parameter, $S$ is the stratification parameter and $Q$ is the heat generation or absorption parameter on the velocity and temperature. The value of the Prandtl number $P r$ is chosen to be 0.7 , which corresponds air. 
The present results are compared with those of Rangi and Ahmad (2012) for the appropriate reduced cases, and found that there is an excellent agreement, as presented in Table 1.

Table 1: Comparison of results $f^{\prime \prime}(0)$ for different values of curvature parameter $\gamma$ with $M=S=Q=\varepsilon=0$

\begin{tabular}{|c|c|c|}
\hline \multirow{2}{*}{$\gamma$} & \multicolumn{2}{|c|}{$f^{\prime \prime}(0)$} \\
\cline { 2 - 3 } & Rangi and Ahmad (2012) & Present Results \\
\hline 0.0 & -1.0000 & -1.00006 \\
\hline 0.25 & -1.094378 & -1.09438 \\
\hline 0.5 & -1.188715 & -1.18869 \\
\hline 0.75 & -1.281833 & -1.28180 \\
\hline 1.0 & -1.459308 & -1.45931 \\
\hline
\end{tabular}

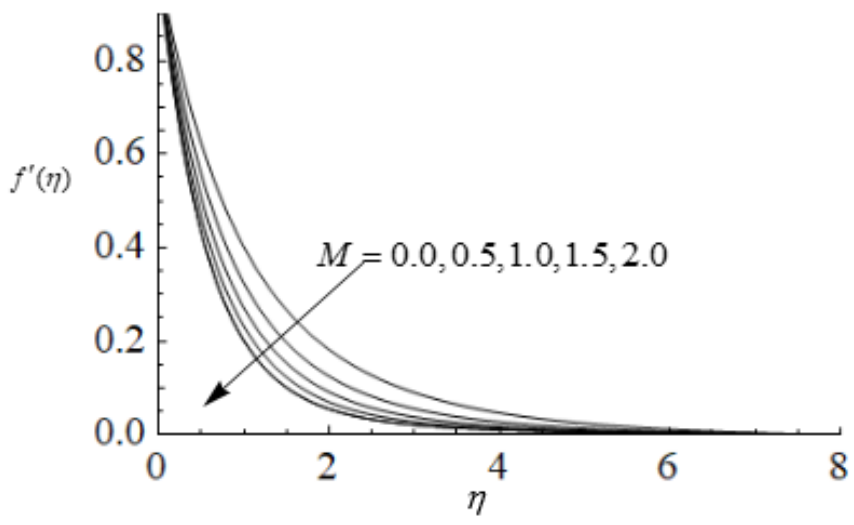

Fig. 2 Velocity profiles for different values of $M$

It is observed from figure 2, that the swiftness of flow gently reduces with the application transverse magnetic field which is due to a resistive type force called Lorentz force similar to drag force which in turn impedes the motion of the fluid flow and thus reducing its velocity. The velocity profiles reaches the free stream velocity at $\eta=7$. The temperature profiles with two cases, constant and varying thermal conductivity (Figure 3 ). For, $\varepsilon=0.1$, the temperature profiles are pronounced than $\varepsilon=0$. Stratification also has significant influence on the temperature profiles.

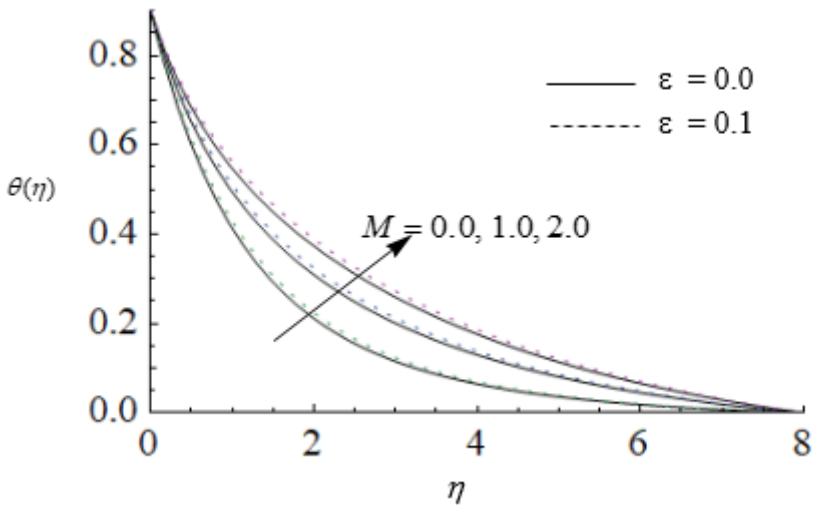

Fig. 3 Temperature profiles for different values of $M$

From figure 4 it is clear that, the velocity of the flow field is reversing phenomena near the region $[0,0.63]$ and at $\eta=0.63$, the profiles coincides then it approaches to zero within the region $[0.63, \infty)$. Velocity profile of the fluid increases with an in increase in the curvature parameter $\gamma$. If $\gamma=0$, the present geometry turns to flat plate.

Figures 6 and 7 show for ascending $\lambda$ values, the buoyancy force becomes stronger, resulting in an increase of both the velocity and temperature profiles. Temperature is found to decrease with an increasing $P r$.

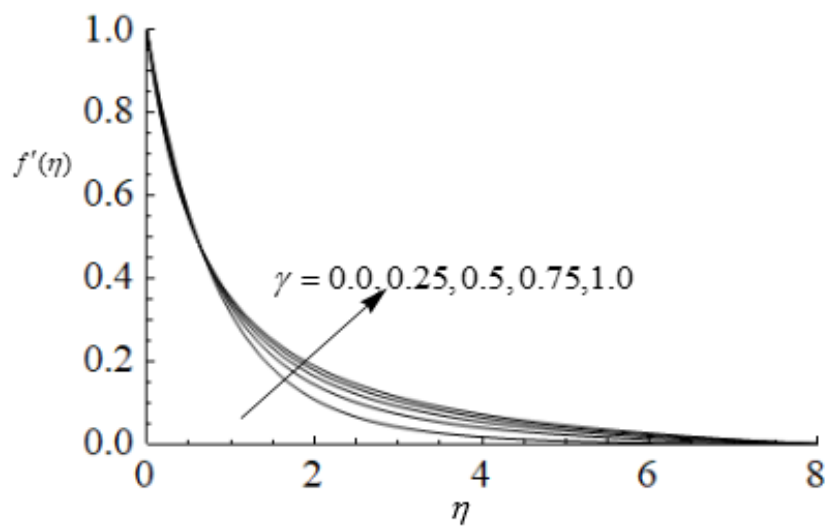

Fig. 4 Velocity profiles for different values of $\gamma$

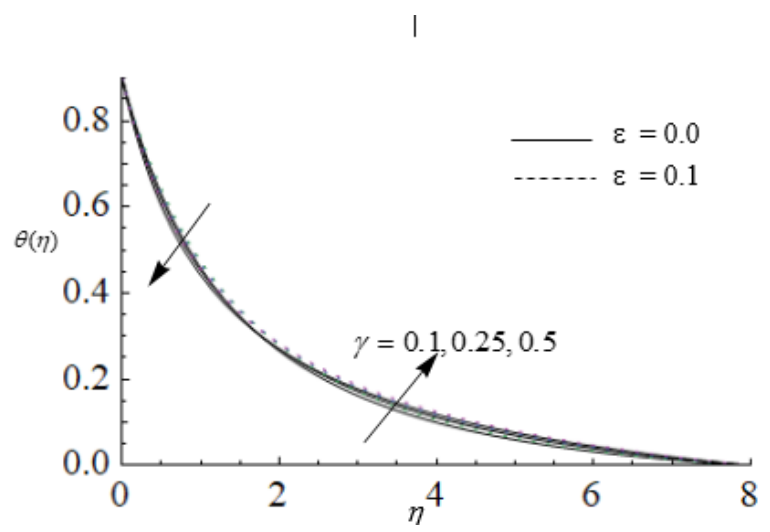

Fig. 5 Temperature profiles for different values of $\gamma$

Liquefied metals possess small Prandtl number usually varies of order $0.01 \sim 0.1$ (from Bismuth (0.01) to Mercury (0.023)) and are preferred as coolants due to its high conductivity of heat. Such fluids have thicker thermal boundary layer. So, heat can diffuse from the surface faster than thinner boundary layers. Hence, Prandtl number controls the cooling rate in conducting flows.

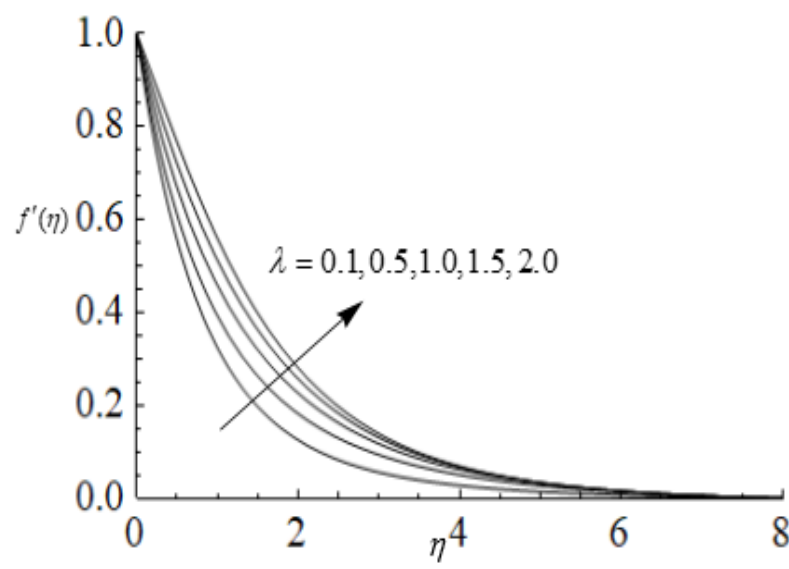

Fig. 6 Velocity profiles for different values of $\lambda$ 
The temperature of the fluid increases with an increase in heat generation or absorption parameter $Q$ (Figure 9). $Q=-0.1$ denotes the heat absorption. Positive values of $Q$ represent heat source. The presence of $\varepsilon$ has a significant impact on the fluid temperature.

Figure 10 depicts that an increase in $S$ results in decrease of the temperature, as the density variation will be less if one goes deeper and deeper inside the lake. Ambient thermal stratification shows considerable decrease the local buoyancy levels, which reduces the motion of the fluid.

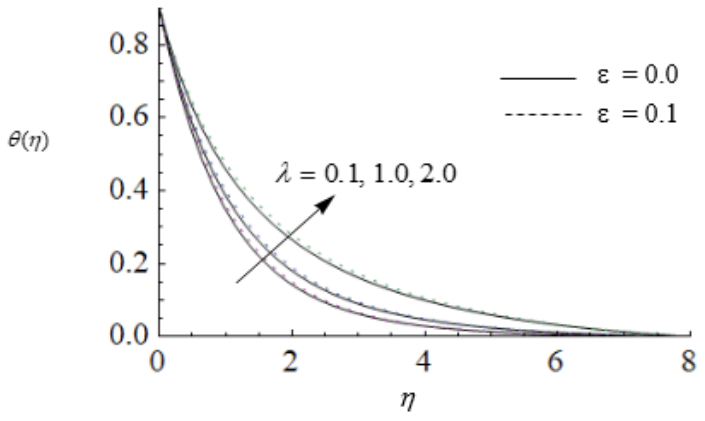

Fig. 7 Temperature profiles for different values of $\lambda$

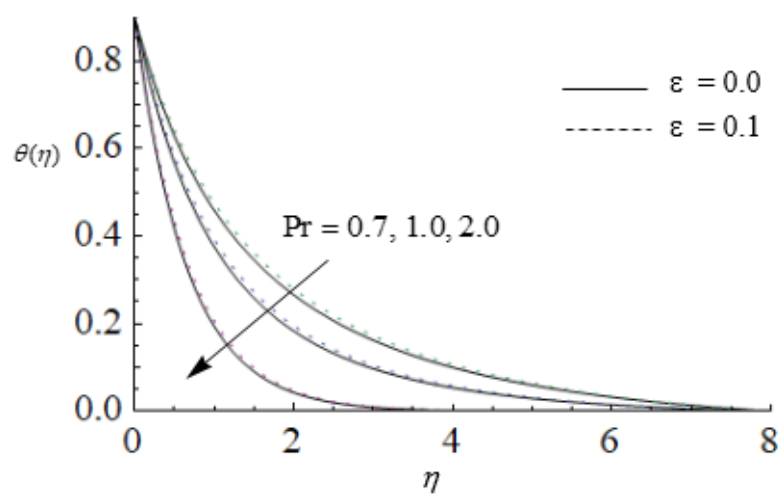

Fig. 8 Temperature profiles for different values of $\mathrm{Pr}$

If the temperature profiles are observed ( Figure 5), the similar trend as like that of velocity can be seen. Initially, the heat in the fluid decreases in the domain $[0,1.63]$ near the surface and increases after that domain up to the free stream surface. Also it is observed that, the transfer of heat the presence of variable thermal conductivity $(\varepsilon=0.1)$ pronounces more than constant thermal conductivity $(\varepsilon=0)$.

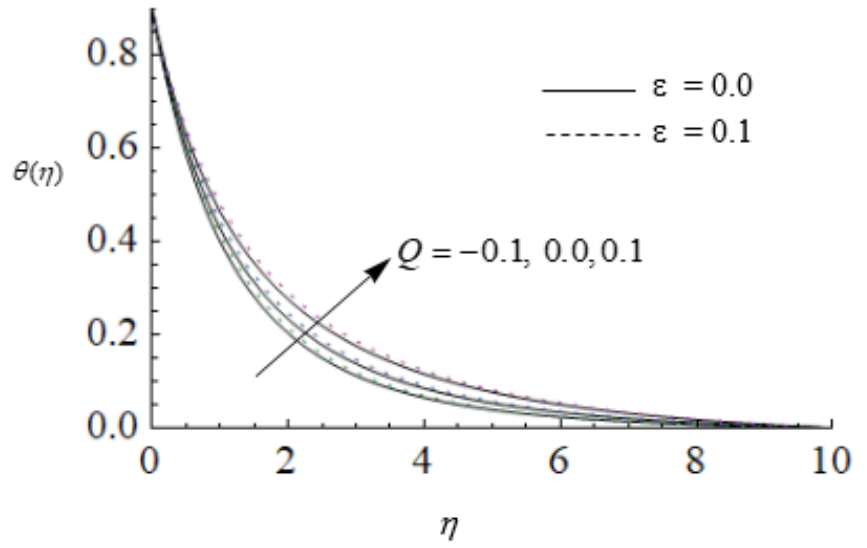

Fig. 9 Temperature profiles for different values of $Q$

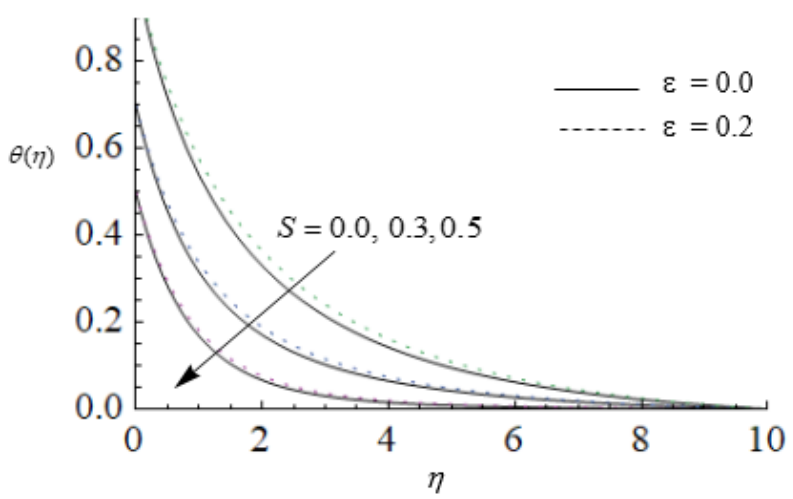

Fig. 10 Temperature profiles for different values of $S$

Figure 11 illustrates the temperature rise with increasing variable thermal conductivity parameter $\varepsilon$ on the temperature. When $\varepsilon=0$, the profile drawn will be with constant thermal conductivity, whereas the profiles rises for varying $\varepsilon$.

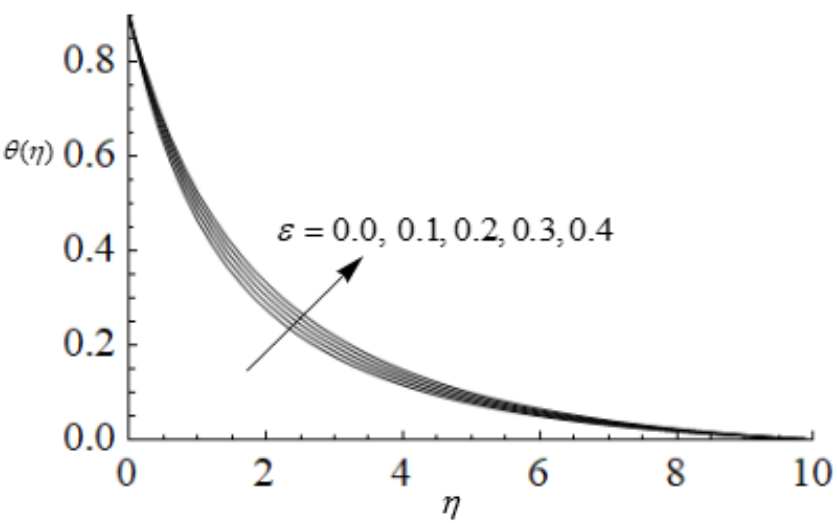

Fig. 11 Temperature profiles for different values of $\varepsilon$

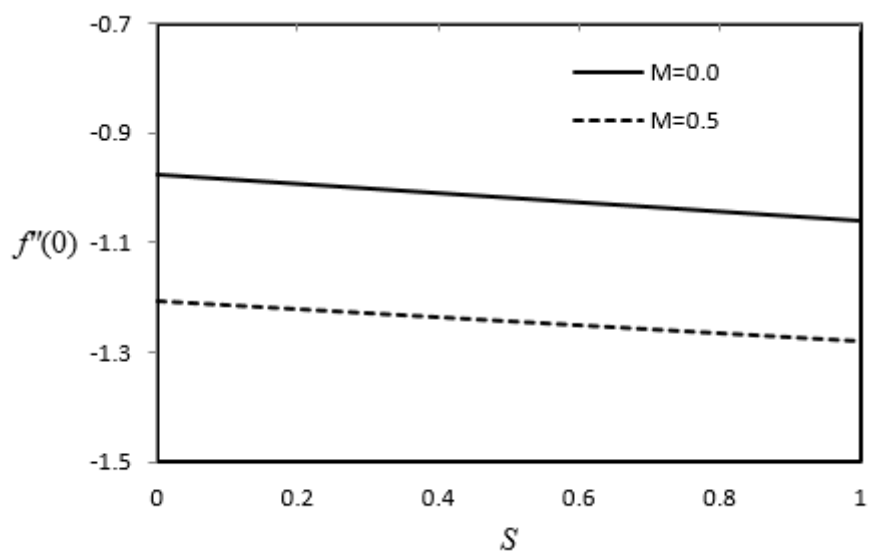

Fig. 12 Surface skinfriction versus $S$ for different $M$

The variations of the velocity and temperature gradient at the surface are proportional to $f^{\prime \prime}(0)$ and - $\theta^{\prime}(0)$. From Figure 12, it is clear that the surface velocity gradient decreases with an increase in $S$.

Also it is observed that the surface skin friction coefficient decreases with an increasing $M$. It is clear that ( Figure 13), the wall shear stress decreases with $S$ and $\gamma$. Also friction is more at the surface of the cylinder $(\gamma=0.5)$ rather than the flat plate $(\gamma=0)$. 


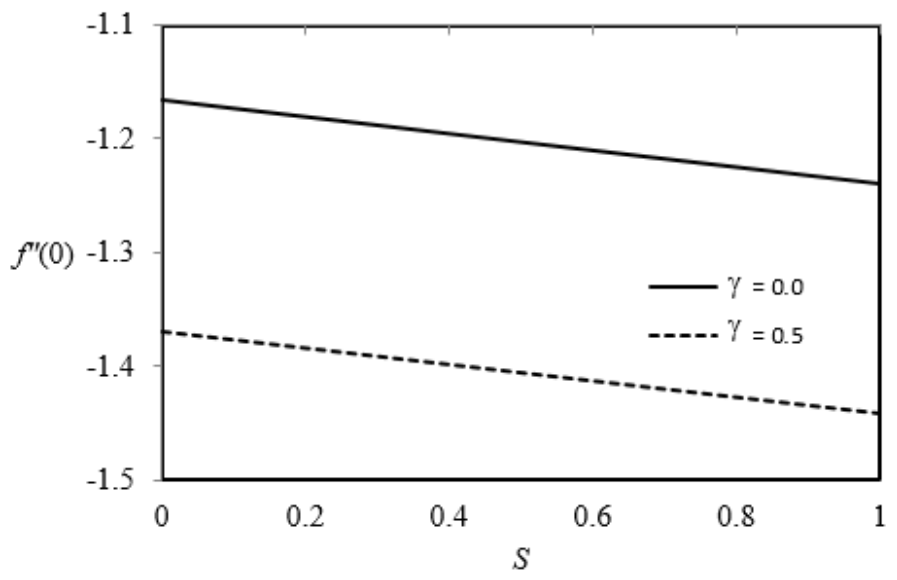

Fig. 13 Surface skinfriction versus $S$ for different values of $\gamma$

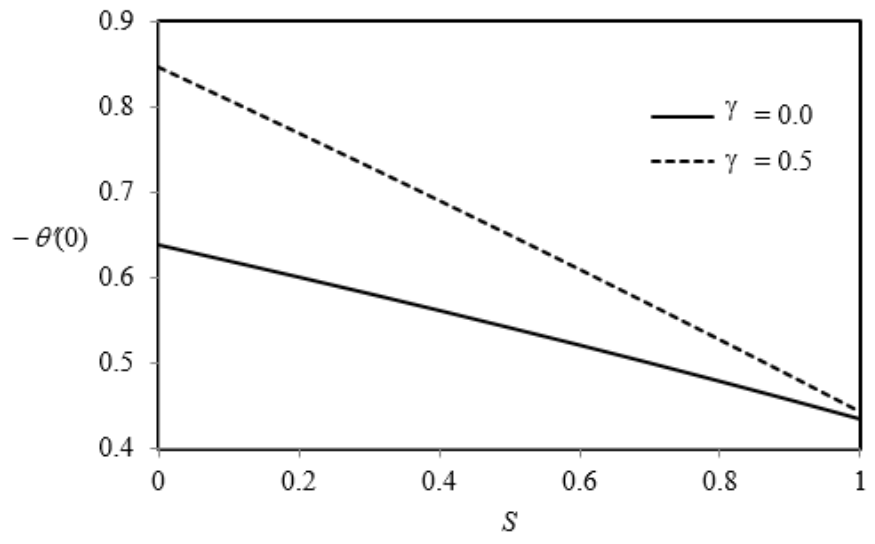

Fig. 14 Heat transfer rate versus $S$ for different values of $\gamma$

The temperature gradient values are less with increasing $S$ and $\gamma$. For higher $\operatorname{Pr}$ values, heat transfer rate is also high.(Figure 15). The rate of heat transfer at the stretching surface decreases with an increase in $S$ for different $Q$ ( Figure 16).

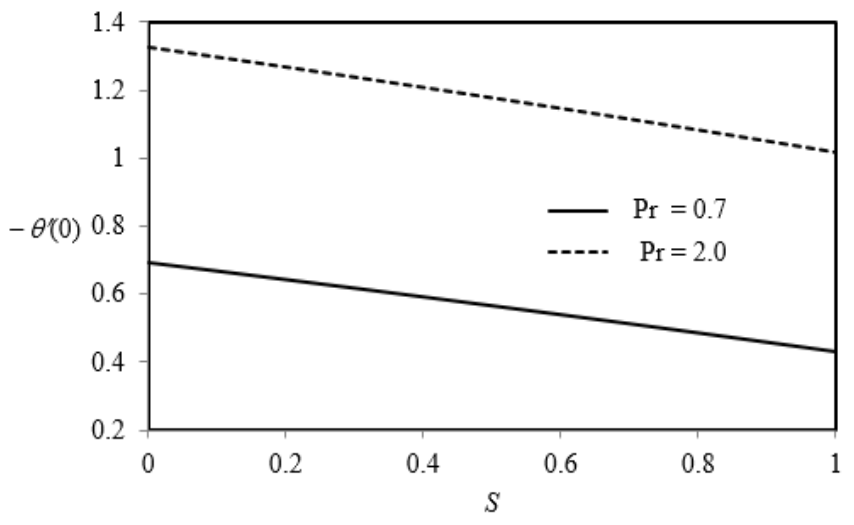

Fig. 15 Heat transfer rate versus $S$ for different values of $P r$

Figure 17 depicts the rate of heat transfer versus stratification parameter $S$, for different $\varepsilon$. It is observed that all profiles are converging at a common point $S=1$ and the temperature gradient is 0.45 .

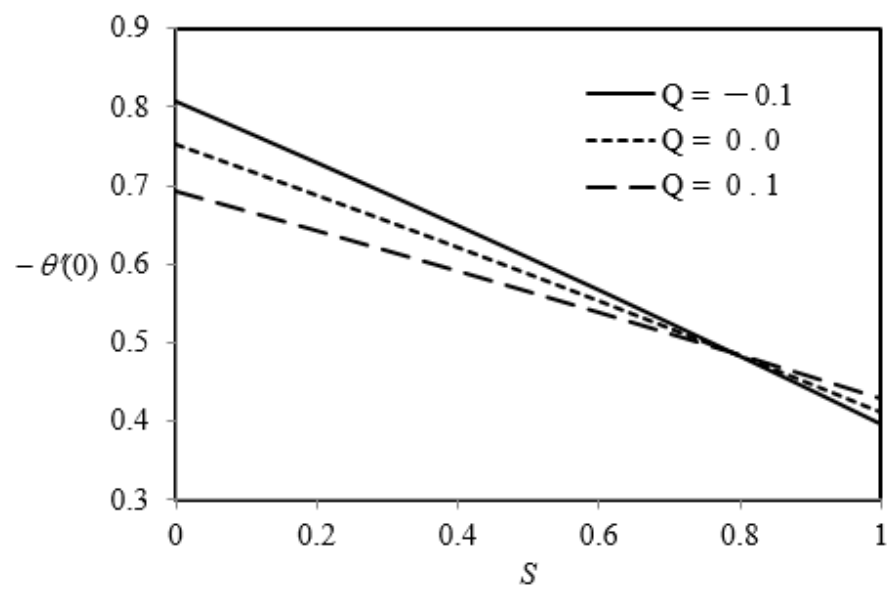

Fig. 16 Heat transfer rate versus $S$ for different values of $Q$

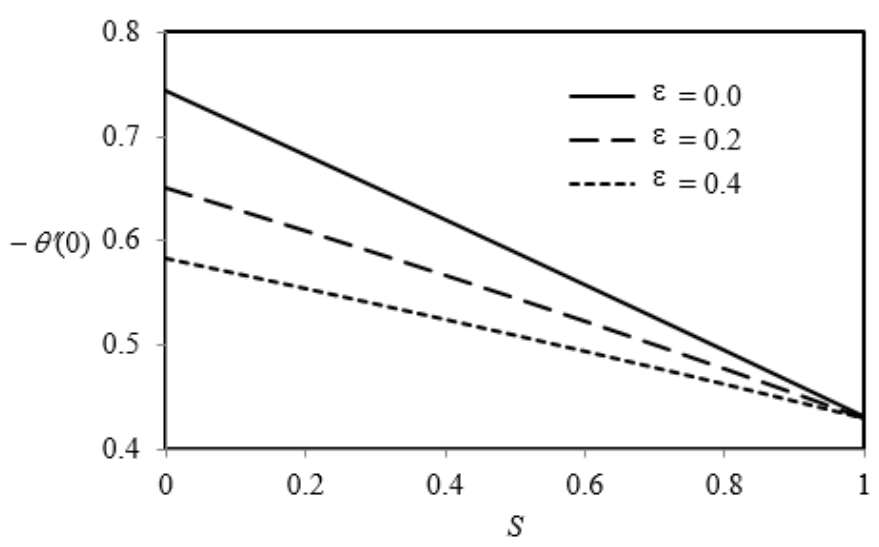

Fig. 17 Heat transfer rate versus $S$ for different values of $\varepsilon$

\section{CONCLUSIONS}

A mathematical model has been framed to examine the influence of variable thermal conductivity on MHD flow past a stretching cylinder imbedded in a thermally stratified medium with internal heat sour or sink. The following conclusions drawn are as follows:

- The curvature of stretching cylinder is an essential parameter, has considerable influence on motion of the flow and temperature field too.

- Due to the wall stretching, the skin friction coefficient decreases for both $\gamma$ and $M$

- Presence of the buoyancy force decreases the magnitude of both the skin friction coefficient and heat transfer rate at the surface as the thermal stratification increases.

- The magnitude of the both the skin friction coefficient and heat transfer rate at the surface are higher for a cylinder compared to a flat plate.

- For varying thermal conductivity, the curvature of the cylinder helps to enhance the heat transfer from the fluid.

\section{NOMENCLATURE}

$B_{0}$ magnetic field

$T_{\infty}$ ambient temperature

$T_{w} \quad$ wall temperature

$\mathrm{R}$ radius of the cylinder 
$u, v$ the velocity components

$x, r$ coordinates

$T$ the temperature of the nanofluid

$T_{\infty}$ the ambient fluid temperature

$U_{0}$ velocity parameter of the stretching surface

$T_{0}$ parameter of the temperature distribution

$L \quad$ the characteristic length

b,c positive constants

$U$ stretching velocity

$C$ heat capacity $\left(\mathrm{J} / \mathrm{m}^{3} \cdot \mathrm{K}\right)$

$c_{p} \quad$ specific heat $(\mathrm{J} / \mathrm{kg} \cdot \mathrm{K})$

$k$ thermal conductivity $(\mathrm{W} / \mathrm{m} \cdot \mathrm{K})$

$g$ gravity

$T_{w} \quad$ prescribed surface temperature and

$\mathrm{T}_{\infty}$ ambient temperature

$M$ the magnetic parameter

$P r$ the Prandtl number

$S$ the stratification parameter

$Q$ the heat generation or absorption parameter

f dimensionless variable

\section{Greek symbols}

$\theta$ dimensionless temperature

$\phi$ dimensionless concentration

$\beta \quad$ thermal coefficient of expansion

$\sigma \quad$ the electric conductivity

$\rho$ the fluid density

$\alpha$ thermal diffusivity

$\mu$ the coefficient of fluid viscosity

$\varepsilon \quad$ the thermal conductivity parameter

$v$ the kinematic viscosity of the base fluid

$\eta \quad$ similarity variable

$\gamma \quad$ the curvature parameter

$\lambda$ the mixed convection parameter

\section{REFERENCES}

Alireza Rasekh, Davood D Ganji, Sady Tavakoli, 2012, "Numerical Solutions for a Nanofluid past over a Stretching Circular Cylinder with Non-Uniform Heat Source," Frontiers in Heat and Mass Transfer, 3, 10 .

https://doi.org/10.5098/hmt.v3.4.3003

Bhaskar Reddy N., Poornima T., and Sreenivasulu P., 2014, “ Influence of Variable Thermal Conductivity on MHD Boundary Layer Slip Flow of Ethylene-Glycol Based Cu Nanofluids over a Stretching Sheet with Convective Boundary Condition," International Journal of Engineering Mathematics, Article ID 905158, 10.

http://dx.doi.org/10.1155/2014/905158.

Dulal Pal and Hiranmoy Mondal, 2010, "Effect of Variable Viscosity on MHD Non-Darcy Mixed Convective Heat Transfer over a Stretching Sheet Embedded in a Porous Medium with Non-Uniform Heat Source/Sink," Communications in Nonlinear Science and Numerical Simulation, 15 (6), 1553-1564.

https://doi.org/10.1016/j.cnsns.2009.07.002

Subba Rao, A., Ramachandra Prasad, V., Harshavalli, K., and Anwer Beg, O., 2016, "Thermal Radiation Effects on Non-Newtonian Fluid in a Variable Porosity Regime with Partial Slip," Journal of Porous Media, 19 (4), 313-329.

https://doi.org/10.1615/JPorMedia.v19.i4.30

Hayat, T., Shafiq, A., Alsaedi, A. and Shahzad, S. A., 2016, "Unsteady MHD Flow over Exponentially Stretching Sheet with
Slip Conditions," Applied Mathematics and Mechanics, 37 (2), 193208.

https://doi.org/10.1007/s10483-016-2024-8

Hayat, T., Tariq Hussain, Shehzad, S. A., and Alsaedi, A., 2014, "Thermal and Concentration Stratifications Effects in Radiative Flow of Jeffrey Fluid over a Stretching Sheet," PLoS ONE , 9(10), e107858, http://dx.doi.org/10.1371/journal.pone.0107858.

Sharma, K., and Sumit Gupta, 2016, "Analytical Study of MHD Boundary Layer Flow and Heat Transfer towards a Porous Exponentially Stretching Sheet in presence of Thermal Radiation," International Journal of Advances in Applied Mathematics and Mechanics, 4(1), 1 - 10.

Kishore, P. M., Rajesh, V., and Vijayakumar Verma, S., 2010, "Thermal Stratification and Viscous Dissipation Effects on MHD Unsteady Radiative Natural Convection Flow past an Infinite Vertical Accelerated Plate Embedded in a Porous Medium with Variable Surface Temperature," Int. J. of Appl. Math and Mech., 6(16), 1-18.

Nirmal Chand Peddisetty, 2016, "Effects of Thermal Stratification on Transient Free Convective Flow of a Nanofluid past a Vertical Plate," Pramana-J. Phys., 87, 62. https://doi.org/10.1007/s12043-016-1266-y

Odelu Ojjela , Kashyap, K. P., Kumar, N. N., and Das, S. K ., 2016, "Unsteady Flow and Heat Transfer of UCM Fluid in a Porous Channel with Variable Thermal Conductivity and Ion Slip Effects," Frontiers in Heat and Mass Transfer, 7 - 013032. http://dx.doi.org/10.5098/hmt.7.32

Poornima, T., Bhaskar Reddy, N., and Sreenivasulu, P., 2015, "Slip Flow of Casson Rheological Fluid under Variable Thermal Conductivity with Radiation," Heat Transfer Asian Research Journal, 44(8), 718-737. https://doi.org/10.1002/htj.21145

Poornima, T., Sreenivasulu, P., and Bhaskar Reddy, N.,2016, "Chemical Reaction Effects on an Unsteady MHD Mixed Convective and Radiative Boundary Layer Flow past a Circular Cylinder," Journal of Applied Fluid Mechanics, 9(6), 2877-2885.

https://doi.org/10.2298/CICEQ101218011A

Rekha Rangi and Naseem Ahmad, 2012, "Boundary Layer Flow past a Stretching Cylinder and Heat Transfer with Variable Thermal Conductivity," Applied Mathematics, 3, 205-209

http://dx.doi.org/10.4236/am.2012.33032.

Rosmila, A. B., Kandasamy R., and Muhaimin I., 2012, "Lie Symmetry Group Transformation For MHD Natural Convection Flow of Nanofluid over Linearly Porous Stretching Sheet in Presence of Thermal Stratification," Applied Mathematics and Mechanics, 33(5), 593-604.

https://doi.org/10.1007/s10483-012-1573-9

Deka, R. K. , and Neog, B. C., 2009, "Unsteady Natural Convection Flow past an Accelerated Vertical Plate in a Thermally Stratified Fluid," Theoret. Appl. Mech., 36(4), 261-274. https://doi.org/10.2298/TAM0904261D

Rudra Kanta Deka and Ashish Paul, 2012, "Transient Free Convection Flow past an Infinite Vertical Cylinder with Thermal Stratification," Journal of Mechanical Science and Technology, 26 (8), 2229-2237. https://doi.org/10.1007/s12206-012-0602-5 
Rushi Kumar, B., Sravan Kumar, T., and Vijaya Kumar, A. G., 2015, "Thermal Diffusion and Radiation Effects on Unsteady Free Convection Flow in the presence of Magnetic Field Fixed Relative to the Fluid or to the Plate," Frontiers in Heat and Mass Transfer, 6 013012.

https://doi.org/10.5098/hmt.6.12

Salahuddin, T., Malik, M. Y., Arif Hussain, Bilal, S. and Awais, M., 2016, "Combined Effects of Variable Thermal Conductivity and MHD Flow on Pseudoplastic Fluid over a Stretching Cylinder by using Keller Box Method," Information Sciences Letters, 5(1), 11-19. http://dx.doi.org/10.18576/isl/050102.

Sreenivasulu, P., and Bhaskar Reddy, N., 2013, "Radiation And Mass Transfer Effects On Boundary Layer Stagnation Point-Flow Of A Nanofluid Over A Permeable Stretching Sheet In A Porous Medium With Heat Absorption/Generation: A Lie Group Analysis," Annals of Mathematical Physics, 5, 61-75.

Sreenivasulu, P., Poornima, T., and Bhaskar Reddy, N., 2015, "Influence Of Magnetic Field On A Nanofluid Past A Non Linear Stretching Sheet With Uniform Heat Source," Atti della Fondazione Giorgio Ronchi,Estratto da: Atti della Fondazione Giorgio Ronchi, LXX (2), Marzo-Aprile 2015.
Subba Rao, A., and Nagendra, N., 2015, "Thermal Radiation Effects on Oldroyd-B Nanofluid from A Stretching Sheet In A Non-Darcy Porous Medium," Global J. Pure and Applied Mathematics, 11, 45-49.

Subhas Abel, M., Siddheshwar, P.G., and Mahesha, N., 2009, "Effects Of Thermal Buoyancy And Variable Thermal Conductivity On The MHD Flow And Heat Transfer In A Power-Law Fluid Past A Vertical Stretching Sheet In The Presence Of A Non-Uniform Heat Source," International Journal of Non-Linear Mechanics, 44 (1), 1-12. https://doi.org/10.1016/j.ijnonlinmec.2008.08.002.

Mukhopadhyay, S., and Ishak, A., 2012, "Mixed Convection Flow along a Stretching Cylinder in a Thermally Stratified Medium," Journal of Applied Mathematics, 2012, Article ID 491695, 8 pages. http://dx.doi.org/10.1155/2012/491695.

Mahapatra,T. R., Sidui, S., and Nandy, S.K., 2014, "Effect of Magnetic Field On Indirect Natural Convection Flow Above A Horizontal Hot Flat Plate," Frontiers in Heat and Mass Transfer, 5 - 013015. https://doi.org/10.5098/hmt.5.15.

Ibrahim, W., and Shankar, B., 2016, "The Effects of Thermal Radiation and Non-Uniform Heat Source/Sink on MHD Boundary-Layer Flow and Heat Transfer past a Stretching Sheet Embedded in Non-Darcian Porous Medium," Frontiers in Heat and Mass Transfer, 7 - 013037. https://doi.org/10.5098/hmt.7.37 